\title{
Erratum: Spin and orbital angular momentum of a class of nonparaxial light beams having a globally defined polarization [Phys. Rev. A 80, 063814 (2009)]
}

\author{
Chun-Fang Li \\ (Received 2 April 2016; published 15 April 2016)
}

DOI: 10.1103/PhysRevA.93.049902

In my work on the spin and orbital angular momentum (OAM) of light, I showed that the spin of a radiation field stems from a movement of the field that contributes nothing to the total (linear) momentum. This was done by expanding the electric and magnetic vectors of a radiation field in terms of their plane-wave spectra in the following way:

$$
\begin{aligned}
& \mathcal{E}(\mathbf{x}, t)=\frac{1}{\sqrt{2}}\left[\frac{1}{(2 \pi)^{3 / 2}} \int \mathbf{E} e^{i \mathbf{k} \cdot \mathbf{x}} d^{3} k+\text { c.c. }\right], \\
& \mathcal{H}(\mathbf{x}, t)=\frac{1}{\sqrt{2}}\left[\frac{1}{(2 \pi)^{3 / 2}} \int \frac{\mathbf{k} \times \mathbf{E}}{\mu_{0} \omega} e^{i \mathbf{k} \cdot \mathbf{x}} d^{3} k+\text { c.c. }\right],
\end{aligned}
$$

where $\mathbf{E}=\mathbf{E}(\mathbf{k}, t)$ is the electric vector in reciprocal space, $\mathbf{k}$ is the wave vector, and $\omega=c|\mathbf{k}|$ is the angular frequency. Of course, the transversality condition on the electric vector $\nabla \cdot \mathcal{E}=0$ means

$$
\mathbf{k} \cdot \mathbf{E}=0 \text {. }
$$

However, considering that the electric and magnetic vectors (1) are both real valued, no conditions other than Eq. (2) should be imposed on $\mathbf{E}$. Therefore, the conditions (5) in my work, which amount to $\mathbf{E}(-\mathbf{k}, t)=\mathbf{E}^{*}(\mathbf{k}, t)$, are redundant. In this Erratum I want to point out that those redundant conditions do not alter, except for a factor of 2, the final expressions for the energy, momentum, and angular momentum in reciprocal space. This fact has already been taken into account by the factor $1 / \sqrt{2}$ in Eqs. (1), which takes the place of the factor $1 / 2$ in Eqs. (3) and (4) of my work. Here are the detailed derivations.

The contribution of the electric field to the total energy is defined [1] as $W_{e}=\frac{\varepsilon_{0}}{2} \int \mathcal{E}^{2} d^{3} x$. Upon substituting Eq. (1a), one finds, by a straightforward calculation,

$$
W_{e}=\frac{\varepsilon_{0}}{2} \int \mathbf{E}^{*} \cdot \mathbf{E} d^{3} k+\frac{\varepsilon_{0}}{4} \int[\mathbf{E}(-\mathbf{k}, t) \cdot \mathbf{E}(\mathbf{k}, t)+\text { c.c. }] d^{3} k .
$$

Similarly, the contribution of the magnetic field to the total energy, which is defined as $W_{m}=\frac{\mu_{0}}{2} \int \mathcal{H}^{2} d^{3} x$, is calculated to be

$$
W_{m}=\frac{\varepsilon_{0}}{2} \int \mathbf{E}^{*} \cdot \mathbf{E} d^{3} k-\frac{\varepsilon_{0}}{4} \int[\mathbf{E}(-\mathbf{k}, t) \cdot \mathbf{E}(\mathbf{k}, t)+\text { c.c. }] d^{3} k .
$$

The expression for the total energy in reciprocal space thus reads

$$
W=W_{e}+W_{m}=\int \varepsilon_{0} \mathbf{E}^{*} \cdot \mathbf{E} d^{3} k .
$$

Next, we look at the momentum. The momentum density in real space is defined as $\mathbf{p}(\mathbf{x}, t)=\varepsilon_{0} \mu_{0} \mathcal{E} \times \mathcal{H}$. Upon substituting Eqs. (1) and with the help of the relation

$$
\mathbf{a} \times(\mathbf{b} \times \mathbf{c})=(\mathbf{a} \cdot \mathbf{c}) \mathbf{b}-(\mathbf{a} \cdot \mathbf{b}) \mathbf{c},
$$

it splits into two parts

$$
\mathbf{p}(\mathbf{x}, t)=\mathbf{p}_{1}(\mathbf{x}, t)+\mathbf{p}_{2}(\mathbf{x}, t),
$$

where

$$
\begin{aligned}
\mathbf{p}_{1} & =\frac{\varepsilon_{0}}{2(2 \pi)^{3}} \int \frac{\mathbf{E}^{*} \cdot \mathbf{E}^{\prime}}{\omega} \mathbf{k} e^{i\left(\mathbf{k}^{\prime}-\mathbf{k}\right) \cdot \mathbf{x}} d^{3} k^{\prime} d^{3} k+\frac{\varepsilon_{0}}{2(2 \pi)^{3}} \int \frac{\mathbf{E} \cdot \mathbf{E}^{\prime}}{\omega} \mathbf{k} e^{i\left(\mathbf{k}^{\prime}+\mathbf{k}\right) \cdot \mathbf{x}} d^{3} k^{\prime} d^{3} k+\text { c.c. } \\
\mathbf{p}_{2} & =-\frac{\varepsilon_{0}}{2(2 \pi)^{3}} \int \frac{\mathbf{k} \cdot \mathbf{E}^{\prime}}{\omega} \mathbf{E}^{*} e^{i\left(\mathbf{k}^{\prime}-\mathbf{k}\right) \cdot \mathbf{x}} d^{3} k^{\prime} d^{3} k-\frac{\varepsilon_{0}}{2(2 \pi)^{3}} \int \frac{\mathbf{k} \cdot \mathbf{E}^{\prime}}{\omega} \mathbf{E} e^{i\left(\mathbf{k}^{\prime}+\mathbf{k}\right) \cdot \mathbf{x}} d^{3} k^{\prime} d^{3} k+\text { c.c. },
\end{aligned}
$$

and $\mathbf{E}^{\prime}=\mathbf{E}\left(\mathbf{k}^{\prime}, t\right)$. Straightforward calculations give for the contribution of the first part of the momentum density to the total momentum

$$
\mathbf{P}_{1}=\int \mathbf{p}_{1} d^{3} x=\int \frac{\varepsilon_{0}}{\omega}\left(\mathbf{E}^{*} \cdot \mathbf{E}+\Delta\right) \mathbf{k} d^{3} k
$$


where

$$
\Delta=\frac{1}{2}\left[\mathbf{E}^{*}(-\mathbf{k}, t) \cdot \mathbf{E}^{*}(\mathbf{k}, t)+\mathbf{E}(-\mathbf{k}, t) \cdot \mathbf{E}(\mathbf{k}, t)\right] .
$$

Because $\Delta$ is an even function of $\mathbf{k}$, one actually has

$$
\mathbf{P}_{1}=\int \frac{\varepsilon_{0} \mathbf{E}^{*} \cdot \mathbf{E}}{\omega} \mathbf{k} d^{3} k .
$$

In addition, it is easy to show, upon taking the transversality condition (2) into account, that the second part of the momentum density contributes nothing to the total momentum,

$$
\mathbf{P}_{2}=\int \mathbf{p}_{2} d^{3} x=0
$$

Therefore, the total momentum assumes the form

$$
\mathbf{P}=\mathbf{P}_{1}=\int \frac{\varepsilon_{0} \mathbf{E}^{*} \cdot \mathbf{E}}{\omega} \mathbf{k} d^{3} k .
$$

At last, we pay attention to the angular momentum about a reference point $\mathbf{x}_{0}$, which is defined as

$$
\mathbf{J}\left(\mathbf{x}_{0}\right)=\int\left(\mathbf{x}-\mathbf{x}_{0}\right) \times \mathbf{p} d^{3} x=\mathbf{J}(0)-\mathbf{x}_{0} \times \int \mathbf{p} d^{3} x,
$$

where

$$
\mathbf{J}(0)=\int \mathbf{x} \times \mathbf{p} d^{3} x
$$

is the angular momentum about the origin. That the momentum density splits into two different parts makes it possible to separate the angular momentum into two distinct parts. One part is associated with the second momentum density $\mathbf{p}_{2}$. Because of the property (6), this part, denoted by $\mathbf{S}$, is independent of the choice of the reference point,

$$
\mathbf{S}\left(\mathbf{x}_{0}\right)=\mathbf{S}(0)=\int \mathbf{x} \times \mathbf{p}_{2} d^{3} x,
$$

and is reasonably regarded as the spin. The other part is associated with the first momentum density $\mathbf{p}_{1}$. Clearly, it depends on the choice of the reference point

$$
\mathbf{L}\left(\mathbf{x}_{0}\right)=\mathbf{L}(0)-\mathbf{x}_{0} \times \mathbf{P}
$$

and is reasonably regarded as the OAM, where

$$
\mathbf{L}(0)=\int \mathbf{x} \times \mathbf{p}_{1} d^{3} x
$$

is the OAM about the origin and $\mathbf{P}$ is the total momentum (7). Let us derive the expressions for the spin (8) and OAM (9) in reciprocal space.

Substituting Eq. (5) into Eq. (8), one has

$$
\mathbf{S}=\mathbf{S}_{1}+\mathbf{S}_{2}+\text { c.c. },
$$

where

$$
\begin{aligned}
& \mathbf{S}_{1}=\frac{\varepsilon_{0}}{2(2 \pi)^{3}} \int \frac{\mathbf{k} \cdot \mathbf{E}^{\prime}}{\omega} \mathbf{E}^{*} \times \mathbf{x} e^{i\left(\mathbf{k}^{\prime}-\mathbf{k}\right) \cdot \mathbf{x}} d^{3} x d^{3} k^{\prime} d^{3} k, \\
& \mathbf{S}_{2}=\frac{\varepsilon_{0}}{2(2 \pi)^{3}} \int \frac{\mathbf{k} \cdot \mathbf{E}^{\prime}}{\omega} \mathbf{E} \times \mathbf{x} e^{i\left(\mathbf{k}^{\prime}+\mathbf{k}\right) \cdot \mathbf{x}} d^{3} x d^{3} k^{\prime} d^{3} k .
\end{aligned}
$$

After performing the integration with respect to $\mathbf{x}, \mathbf{S}_{1}$ changes into

$$
\begin{aligned}
\mathbf{S}_{1}= & -\frac{i \varepsilon_{0} \mathbf{e}_{1}}{2} \int \frac{\mathbf{k} \cdot \mathbf{E}^{\prime}}{\omega} \delta\left(k_{1}^{\prime}-k_{1}\right)\left[E_{2}^{*} \delta\left(k_{2}^{\prime}-k_{2}\right) \delta^{\prime}\left(k_{3}^{\prime}-k_{3}\right)-E_{3}^{*} \delta\left(k_{3}^{\prime}-k_{3}\right) \delta^{\prime}\left(k_{2}^{\prime}-k_{2}\right)\right] d^{3} k^{\prime} d^{3} k \\
& -\frac{i \varepsilon_{0} \mathbf{e}_{2}}{2} \int \frac{\mathbf{k} \cdot \mathbf{E}^{\prime}}{\omega} \delta\left(k_{2}^{\prime}-k_{2}\right)\left[E_{3}^{*} \delta\left(k_{3}^{\prime}-k_{3}\right) \delta^{\prime}\left(k_{1}^{\prime}-k_{1}\right)-E_{1}^{*} \delta\left(k_{1}^{\prime}-k_{1}\right) \delta^{\prime}\left(k_{3}^{\prime}-k_{3}\right)\right] d^{3} k^{\prime} d^{3} k \\
& -\frac{i \varepsilon_{0} \mathbf{e}_{3}}{2} \int \frac{\mathbf{k} \cdot \mathbf{E}^{\prime}}{\omega} \delta\left(k_{3}^{\prime}-k_{3}\right)\left[E_{1}^{*} \delta\left(k_{1}^{\prime}-k_{1}\right) \delta^{\prime}\left(k_{2}^{\prime}-k_{2}\right)-E_{2}^{*} \delta\left(k_{2}^{\prime}-k_{2}\right) \delta^{\prime}\left(k_{1}^{\prime}-k_{1}\right)\right] d^{3} k^{\prime} d^{3} k .
\end{aligned}
$$


Then upon performing the integration with respect to $\mathbf{k}^{\prime}$, one gets

$$
\mathbf{S}_{1}=\frac{i}{2} \int \frac{\varepsilon_{0}}{\omega}\left(\mathbf{E}^{*} \times \nabla_{\mathbf{k}} \mathbf{E}\right) \cdot \mathbf{k} d^{3} k .
$$

Since $\left(\nabla_{\mathbf{k}} \mathbf{E}\right) \cdot \mathbf{k}=-\mathbf{E}$ in accordance with the transversality condition (2), the above expression becomes

$$
\mathbf{S}_{1}=\int \frac{\varepsilon_{0}}{2 i \omega} \mathbf{E}^{*} \times \mathbf{E} d^{3} k
$$

which is obviously real valued. In addition, a similar calculation gives

$$
\mathbf{S}_{2}=\int \frac{i \varepsilon_{0}}{2 \omega} \mathbf{E}(-\mathbf{k}, t) \times \mathbf{E}(\mathbf{k}, t) d^{3} k
$$

which is equal to zero because the integrant is an odd function of $\mathbf{k}$. As a result, the spin takes the following form in reciprocal space:

$$
\mathbf{S}=\int \frac{\varepsilon_{0}}{i \omega} \mathbf{E}^{*} \times \mathbf{E} d^{3} k
$$

Substituting Eq. (4) into Eq. (9), one has

$$
\mathbf{L}(0)=\mathbf{L}_{1}+\mathbf{L}_{2}+\text { c.c. }
$$

where

$$
\begin{aligned}
& \mathbf{L}_{1}=-\frac{\varepsilon_{0}}{2(2 \pi)^{3}} \int \frac{\mathbf{E}^{*} \cdot \mathbf{E}^{\prime}}{\omega} \mathbf{k} \times \mathbf{x} e^{i\left(\mathbf{k}^{\prime}-\mathbf{k}\right) \cdot \mathbf{x}} d^{3} x d^{3} k^{\prime} d^{3} k, \\
& \mathbf{L}_{2}=-\frac{\varepsilon_{0}}{2(2 \pi)^{3}} \int \frac{\mathbf{E} \cdot \mathbf{E}^{\prime}}{\omega} \mathbf{k} \times \mathbf{x} e^{i\left(\mathbf{k}^{\prime}+\mathbf{k}\right) \cdot \mathbf{x}} d^{3} x d^{3} k^{\prime} d^{3} k
\end{aligned}
$$

After performing the integration with respect to $\mathbf{x}, \mathbf{L}_{1}$ becomes

$$
\begin{aligned}
\mathbf{L}_{1}= & \frac{i \varepsilon_{0} \mathbf{e}_{1}}{2} \int \frac{\mathbf{E}^{*} \cdot \mathbf{E}^{\prime}}{\omega} \delta\left(k_{1}^{\prime}-k_{1}\right)\left[k_{2} \delta\left(k_{2}^{\prime}-k_{2}\right) \delta^{\prime}\left(k_{3}^{\prime}-k_{3}\right)-k_{3} \delta\left(k_{3}^{\prime}-k_{3}\right) \delta^{\prime}\left(k_{2}^{\prime}-k_{2}\right)\right] d^{3} k^{\prime} d^{3} k \\
& +\frac{i \varepsilon_{0} \mathbf{e}_{2}}{2} \int \frac{\mathbf{E}^{*} \cdot \mathbf{E}^{\prime}}{\omega} \delta\left(k_{2}^{\prime}-k_{2}\right)\left[k_{3} \delta\left(k_{3}^{\prime}-k_{3}\right) \delta^{\prime}\left(k_{1}^{\prime}-k_{1}\right)-k_{1} \delta\left(k_{1}^{\prime}-k_{1}\right) \delta^{\prime}\left(k_{3}^{\prime}-k_{3}\right)\right] d^{3} k^{\prime} d^{3} k \\
& +\frac{i \varepsilon_{0} \mathbf{e}_{3}}{2} \int \frac{\mathbf{E}^{*} \cdot \mathbf{E}^{\prime}}{\omega} \delta\left(k_{3}^{\prime}-k_{3}\right)\left[k_{1} \delta\left(k_{1}^{\prime}-k_{1}\right) \delta^{\prime}\left(k_{2}^{\prime}-k_{2}\right)-k_{2} \delta\left(k_{2}^{\prime}-k_{2}\right) \delta^{\prime}\left(k_{1}^{\prime}-k_{1}\right)\right] d^{3} k^{\prime} d^{3} k
\end{aligned}
$$

When the integration with respect to $\mathbf{k}^{\prime}$ is performed, it reduces to

$$
\mathbf{L}_{1}=\int \frac{\varepsilon_{0}}{2 i \omega} \mathbf{E}^{\dagger}\left(\mathbf{k} \times \nabla_{\mathbf{k}}\right) \mathbf{E} d^{3} k
$$

where $\mathbf{E}^{\dagger}\left(\mathbf{k} \times \nabla_{\mathbf{k}}\right) \mathbf{E}$ stands for $\left[\mathbf{k} \times\left(\nabla_{\mathbf{k}} \mathbf{E}\right)\right] \cdot \mathbf{E}^{*}$. On the other hand, when the integration with respect to $\mathbf{k}$ is performed, it reduces to, with the replacement $\mathbf{k}^{\prime} \rightarrow \mathbf{k}$,

$$
\mathbf{L}_{1}=\int \frac{i \varepsilon_{0}}{2 \omega}\left[\mathbf{E}^{T}\left(\mathbf{k} \times \nabla_{\mathbf{k}}\right) \mathbf{E}^{*}-\frac{\mathbf{E}^{*} \cdot \mathbf{E}}{\omega}\left(\mathbf{k} \times \nabla_{\mathbf{k}} \omega\right)\right] d^{3} k
$$

Because $\mathbf{k} \times \nabla_{\mathbf{k}} \omega=0$, one has

$$
\mathbf{L}_{1}=\int \frac{i \varepsilon_{0}}{2 \omega} \mathbf{E}^{T}\left(\mathbf{k} \times \nabla_{\mathbf{k}}\right) \mathbf{E}^{*} d^{3} k
$$

A comparison with Eq. (11) reveals that $\mathbf{L}_{1}=\mathbf{L}_{1}^{*}$. Furthermore, a similar calculation shows that $\mathbf{L}_{2}=-\mathbf{L}_{2}$, so $\mathbf{L}_{2}=0$. It is hence concluded that the OAM about the origin assumes the form

$$
\mathbf{L}(0)=\int \frac{\varepsilon_{0}}{i \omega} \mathbf{E}^{\dagger}\left(\mathbf{k} \times \nabla_{\mathbf{k}}\right) \mathbf{E} d^{3} k
$$

in reciprocal space.

[1] L. Mandel and E. Wolf, Optical Coherence and Quantum Optics (Cambridge University Press, New York, 1995), Chap. 10. 